\title{
Effets des stupéfiants sur la conduite automobile
}

\section{Effects of narcotic drugs on driving}

Jean-Pierre ANGER

Laboratoire de Toxicologie, Faculté des Sciences Pharmaceutiques, Université de RENNES 1, 2, Avenue du Professeur Léon Bernard - 35043 RENNES Cedex

* Auteur à qui adresser la correspondance : Jean-Pierre ANGER, Laboratoire de Toxicologie , Faculté des Sciences Pharmaceutiques, Université de RENNES 1, 2, Avenue du Professeur Léon Bernard - 35043 RENNES Cedex

Tél : 0223234867 - E-mail : anger@univ-rennes1.fr

(Reçu le 25 avril 2003 ; accepté le 5 mai 2003)

\section{RÉSUMÉ}

Après absorption par différentes voies (pulmonaire, intranasale, orale ou parentérale), les psychotropes illicites (cannabis, cocaïne, opiacés et amphétamines) franchissent la barrière hémato-encéphalique et gagnent le cerveau dont ils vont perturber le fonctionnement en mimant l'action ou en bloquant la sécrétion ou enfin en empêchant la recapture de certains neuromédiateurs. Ainsi les opiacés inhibent sélectivement de nombreuses activités neuronales induites par des stimulis excitateurs si bien que l'information arrive au cerveau non seulement "en retard " mais considérablement amoindrie, voire déformée et les réactions sont également diminuées. La cocaïne et les amphétamines sont à l'inverse de puissants psychostimulants. Ces composés inhibent, au niveau cérébral la recapture des amines biogènes (noradrénaline, dopamine, sérotonine) ce qui induit un accroissement de la neurotransmission. Sous leur influence, la vigilance augmente, le temps de réaction s'améliore. Cependant d'autres effets associés comme l'euphorie, l'agressivité, la fatigue liée à l'insomnie conduisent à des comportements parfois irrationnels et dangereux. Le cannabis entraîne une désinhibition, un état de somnolence avec détérioration de la perception temporelle et spatiale.

D'une façon générale, le dysfonctionnement cérébral induit par la prise de ces stupéfiants modifie plus ou moins profondément le comportement du conducteur qui ne sera plus en mesure de juger sainement une situation critique et pourra, en conséquence, soit sous estimer le risque ou au contraire aura tendance à augmenter la prise de risque et dans certaines circonstances à favoriser l'accident. MOTS-CLÉS

Stupéfiants, conduite automobile.

\section{SUMMARY}

After absorption by various routes (pulmonary, intra-nasal, oral or parenteral) psychotropic drugs (cannabis, cocaine, opiates and amphetamines) cross the blood-brain barrier, reach the brain and disrupt its functions by mimicking the action, or by blocking secretion or at least by preventing the recapture of some neuromediators. Thus, as opiates selectively inhibit many neuronal activities induced by excitation stimuli, the information that gets to the brain is not only delayed but considerably weakened. The cocaine and amphetamines are to reverse of powerful psychostimulants. These compounds inhibit recapture in the brain of biogen amines (adrenaline, dopamine, serotonin), which induces increased neurotransmission. Under their influence, vigilance increases and reaction time is shorter. However other associated effects such as euphoria, aggressiveness and tiredness linked to insomnia, sometimes lead to dangerous behaviours. Cannabis induces desinhibition, drowsiness, and an alteration of temporal and spatial perception.

In general brain dysfunction induced by taking these drugs has a more or less profound effect on the driver's behaviour. He will no longer be able to assess a critical situation soundly and will, as a result, either underestimate risks or on the contrary have a tendency to take more risks and in some circumstances that may increase the risk of accidents.

\section{KEY-WORDS}

Narcotic drugs, impaired driving. 


\section{Introduction}

Les stupéfiants sont des psychotropes ou substances psycho-actives illicites regroupant essentiellement quatre groupes de produits actuellement recherchés en cas d'accident mortel de la circulation routière : le cannabis, les opiacés, la cocaïne et les amphétamines. Ces substances sont capables de modifier le fonctionnement du cerveau et de générer une dépendance. Bien que d'une ampleur encore mal définie par rapport au problème de l'alcool au volant, on reconnaît aujourd'hui que la conduite d'un véhicule sous l'influence de psychotropes constitue un risque majeur de survenue d'accidents de la circulation routière. Ce constat est attesté par les résultats de nombreux travaux effectués dans ce domaine (1). Des progrès importants réalisés ces dernières années dans la compréhension des effets neuropharmacologiques des psychotropes montrent en effet que l'usage de ces substances est responsable de dysfonctionnements neuronaux ayant pour conséquence une altération significative de l'aptitude à conduire un véhicule en toute sécurité (2). Cette dangerosité est également objectivée par les tests psychomoteurs, les études sur simulateurs de conduite, les tests en situation réelle, les études épidémiologiques ainsi que par l'analyse toxicologique des prélèvements biologiques. Dans cet article très général, nous nous limiterons à l'étude des principaux effets pharmacologiques et psychiques des stupéfiants sur l'aptitude à conduire un véhicule automobile et tenterons ainsi de mieux comprendre les risques qu'ils peuvent occasionner.

\section{Interaction des drogues toxicomanogènes avec la neurotransmission}

Le cerveau constitue un ensemble complexe de circuits neuronaux qui s'organisent en réseaux pour traiter les entrées sensorielles, les relayer jusqu' au cortex puis les traduire en activités comportementales ou psychiques. Les recherches en neurobiologie et en neurochimie ont permis de mieux comprendre l'action des psychotropes sur le système nerveux central et ainsi de localiser des structures cérébrales particulières au sein desquelles l'action des psychotropes produit des modifications de la cinétique de transmission de l'information entre les cellules cérébrales (3). Les cinquante milliards de neurones du cerveau sont reliés les uns aux autres pour transmettre l'information sur le plan fonctionnel mais non sur le plan de la continuité anatomique. L'information est transmise le long du neurone comme une impulsion électrique, activité qui est peu affectée par la plupart des drogues toxicomanogènes. La connexion fonctionnelle entre les neurones est la synapse, lieu de largage des neurotransmetteurs qui acheminent l'information d'un neurone à l'autre. On connaît aujourd'hui une quarantaine de neuromédiateurs différents dans le cerveau. Certains stimulent l'activité cérébrale : ce sont les neurotransmetteurs de type adrénergique : noradrénaline (NA), dopamine (DA), sérotonine (5-HT), glutamate... D'autres neurotransmetteurs au contraire l'atténuent : c'est le cas de l'acide gamma amino butyrique (GABA) ou des endorphines. Chacun de ces neuromédiateurs produit par un type particulier de neurone peut atteindre d'autres neurones et s'y fixer par l'intermédiaire de récepteurs. On peut comparer le neuromédiateur à une clé qui reconnait une serrure particulière, le récepteur, et l'ouvre. Chaque neuromédiateur possède un ou plusieurs récepteurs spécifiques. Ce système permet donc la coexistence d'une grande variété de circuits de communication cérébraux qui régissent notre comportement (humeur, faim, soif, sexualité, agressivité ou passivité, etc.). En temps normal, tous ces neuromédiateurs sont secrétés en quantité infime de telle sorte que l'ensemble de notre comportement se trouve dans un état d'équilibre harmonieux : c'est le bien-être ou état d'homéostasie cérébrale.

Les drogues sont capables d'ouvrir ces serrures biochimiques et de bouleverser cet équilibre soit en mimant l'action, soit en bloquant la sécrétion ou enfin en empêchant la recapture de certains neurotransmetteurs.

Ce disfonctionnement neuronal va gagner deux zones particulièrement réactives du cerveau profond $(4,5)$ :

1. l'hypothalamus (système limbique ou «cerveau des émotions»), centre d'intégration des sensations de satisfaction également impliqué dans les fonctions indispensables à la survie de l'espèce (faim, soif, sexualité) ;

2. l'aire tegmentale ventrale (ATV ou système de récompense), source de production de dopamine dans le cerveau. La dopamine libérée stimule à son tour le noyau accumbens, aujourd'hui reconnu comme le centre du plaisir et de la récompense. Cette stimulation est donc à l'origine d'une récompense mais aussi de dépendance.

Le désir de consommer des drogues serait donc lié au plaisir qu'elles procurent et au souvenir de cet événement agréable! Cette constatation est née des observations faites dans les années 90 sur des modèles animaux comme le rat ou le singe qui, placés dans certaines conditions, ont tendance à répéter la consommation de substances psychoactives. Grâce aux techniques de micro-dialyse qui permettent, à l'aide de micro-électrodes de mesurer et ainsi de connaître les variations de 
concentration en messagers chimiques au niveau d'une zone cérébrale précise, on a découvert l'existence d'un mécanisme d'action commun à tous les psychotropes (alcool, cannabis, nicotine, morphine, amphétamines), caractérisé par l'augmentation du taux de DA au niveau du noyau accumbens. Rien à présent ne nous interdit d'extrapoler à l'homme ces observations chez l'animal et si l'on comprend mieux aujourd'hui comment les drogues agissent sur notre cerveau et par la même sur notre comportement, on conçoit également qu'elles puissent exercer des effets notables sur l'aptitude à conduire un véhicule automobile qui requière attention, vigilance et rapidité d'exécution.

\section{Les drogues et leurs modes d'action}

Bien que toutes les drogues stimulent le circuit de la récompense, il faut noter que les taux de libération de la dopamine sont très différents d'un produit à l'autre. Ceci suppose que les mécanismes initiaux mis en jeu portant sur la libération ou la recapture des neuromédiateurs ou encore sur l'activité électrique des neurones, est forcément différent. Enfin il faut tenir compte également de la vulnérabilité de chaque sujet qui dépend elle aussi de facteurs génétiques et environnementaux, ce qui modifie par là même la réponse du sujet par rapport à une même dose de produit. Même si les différentes drogues toxicomanogènes présentent une communauté d'action sur les taux extracellulaires de dopamine, les observations cliniques montrent clairement que chaque groupe de substances entraîne des effets qui lui sont propres et il importe donc de tracer les grandes lignes de leurs différences (3):

\section{Les psychostimulants (amphétamines, cocaïne)}

Ces substances entraînent une augmentation extracellulaire très importante de DA et de NA par des mécanismes sensiblement différents : les amphétamines chassent les catécholamines de leurs vésicules de stockage ; la cocaïne en bloque la recapture ainsi que celle de la $5 \mathrm{HT}$.

L'amphétamine est le chef de file d'une famille de substances chimiquement et pharmacologiquement proches les unes des autres et dont les effets sont identiques et voisins de ceux induits par l'usage de la cocaïne.

La métamphétamine est la molécule la plus puissante de ce groupe. Elle est commercialisée sous la dénomination «ice» ou «glass» du fait de l'aspect blanc transparent de ses cristaux et de «crank» ou de «crystal» pour la forme salifiée, très hydrosoluble. Il s'agit d'une drogue utilisée sous forme de prise ou injectée (forme salifiée), parfois inhalée dans une pipe ou une cigarette. De nombreuses amphétamines ont été commercialisées comme médicaments stimulants dans les années 70 et certaines spécialités sont demeurées célèbres : Ortédrine ${ }^{\circledR}$, Maxiton ${ }^{\circledR}$, Benzédrine ${ }^{\circledR}$. Des dérivés un peu moins nombreux ont été commercialisés comme anorexigènes : Cafilon ${ }^{\circledR}$, Fringalor ${ }^{\circledR}$. Leur usage a souvent donné lieu à des abus et à des troubles comportementaux en utilisation chronique ; il est désormais strictement contrôlé.

La cocaïne est un alcaloïde extrait des feuilles de coca. Elle existe le plus souvent sous forme de chlorhydrate soluble dans l'eau (neige).Son absorption peut se faire par voie nasale (sniffing) ou plus rarement intraveineuse. Elle existe également sous forme de base, insoluble dans l'eau mais soluble dans les lipides : c'est le crack qui est fumé et qui passe ainsi très rapidement du poumon dans le cerveau. L'utilisation de cette forme de cocaïne induit une dépendance rapide, plus forte que lorsque l'alcaloïde est simplement sniffé.

Dans les pays occidentaux, la cocaïne est essentiellement utilisée par les voies parentérale et pulmonaire. L'injection intraveineuse développe un effet euphorisant en moins de une minute et qui persiste environ un quart d'heure. L'inhalation de la drogue permet l'absorption de la cocaïne par la muqueuse nasale, sans passage pulmonaire mais l'activité de la drogue par cette voie est très réduite du fait d'un effet de premier passage hépatique détruisant 70 à $80 \%$ de la dose. En revanche l'inhalation des vapeurs de crack détermine des effets comparables à ceux de l'injection intraveineuse.

Quelle que soit la voie d'administration, la cocaïne est rapidement dégradée au niveau hépatique et par des estérases plasmatiques en plusieurs métabolites éliminés par voie urinaire : principalement la benzoylecgonine et la méthylecgonine. La demi-vie d'élimination et donc d'action de la drogue est courte : de 30 minutes à 1 heure 30 .

\section{Les opiacés et les narcotiques (héroïne, morphine, codéine, etc.)}

On regroupe sous le terme d'opiacés différents produits remarquables par la puissance de leur action pharmacologique. Ces produits sont soit des alcaloïdes naturels de l'opium (morphine, codéine), soit des dérivés hémi-synthétiques (héroïne, codéthyline, pholcodine), soit enfin des composés totalement synthétiques (buprénorphine, fentanyl). L'emploi et la délivrance de ces substances peuvent être libres de prescription (codéine, codéthyline, pholcodine) ou soumis à la réglementation des stupéfiants (morphine, buprénorphine, méthadone) ou enfin illicite (hérö̈ne). 
Les opiacés inhibent par l'intermédiaire de récepteurs spécifiques $(\mu)$ l'activité des neurones GABAergiques qui eux-mêmes limitent l'activité électrique des cellules dopaminergiques localisées dans l'ATV. Les opiacés diminuent également l'activité électrique des neurones noradrénergiques. Ils possèdent donc un effet clinique différent de celui des psychostimulants caractérisé par une baisse des facultés de traitement des événements extérieurs avec, éventuellement l'apparition de sommeil.

Sur le plan toxicocinétique, rappelons que l'héroïne a une demi-vie sanguine très courte et qu'elle est très rapidement métabolisée en 6-monoacétylmorphine (6MAM) puis en morphine qui sera éliminée principalement sous forme de glucuroconjugué durant plusieurs jours. La codéine et la codéthyline sont également transformées en morphine. La buprénorphine et la méthadone ne se dégradent pas en morphine.

\section{Les entactogènes (MDMA ou ecstasy, $M D A, M B D B, 4-M T A$, etc.)}

L'ecstasy ou méthylènedioxymétamphétamine (MDMA) est une phényléthylamine dont l'usage comme psychostimulant ne cesse de se développer. L'ecstasy est vendue sous forme de comprimés ou de gélules contenant quelques $\mathrm{mg}$.à plus de $200 \mathrm{mg}$ de MDMA. D'autres produits apparentés sont également vendus sous la dénomination d'ecstasy : MDB, MDEA, MBDB,2-CB, etc. contenant parfois diverses substances plus ou moins toxiques.

Tous ces produits sont intermédiaires entre les psychostimulants et les hallucinogènes. Ils entraînent une forte libération de sérotonine parfois associée à une libération faible ou nulle de dopamine.

\section{Le cannabis}

La principale molécule active est le $\Delta$-9-tétrahydrocannabinol $(\Delta-9-\mathrm{THC})$. Des récepteurs au THC (CB1) ont été identifiés entre autres dans le mésencéphale, à proximité des neurones dopaminergiques et leur stimulation diminue la libération de GABA, ce qui contribue à une légère activation dopaminergique. C'est sans doute par ce mécanisme que les récepteurs au THC interviennent sur la motivation. Il existe aussi d'autres récepteurs $\mathrm{CB} 1$ dans l'hippocampe et le cervelet, deux régions susceptibles d'intervenir dans les fonctions de mémorisation et d'équilibre.

\section{Les drogues et leurs effets}

\section{Psychostimulants}

Les effets psychophysiologiques de la consommation de cocaïne résultent pour l'essentiel d'une stimulation intense du système nerveux sympathique. Ils sont variables selon le mode de consommation (6).

L'utilisateur occasionnel ressent constamment les effets psychologiques d'euphorie, de bien-être, d'empathie et d'hypervigilance. Son activité psychique est accrue. Il est sujet à des insomnies. Lorsque les doses sont répétées sur une brève période, la fin de l'activité du produit se traduit par une anxiété inclinant à utiliser de nouveau la drogue. Si la dose utilisée est plus importante, le consommateur peut être en proie à une agitation psychomotrice intense accompagnée d'idées délirantes (sentiment de persécution, illusions sensorielles, amnésies). Des comportements violents ont été rapportés après injection de la drogue ou inhalation de crack. À la longue le consommateur devient sujet à une grande instabilité caractérielle (dysphorie) avec délire d'interprétation à forme paranoïde, attaques de panique, etc. associés à des troubles de la vigilance et de la concentration peu propices à une conduite sereine.

Les amphétamines réduisent le sommeil ou souvent l'empêchent totalement. Elles augmentent de façon temporaire la vigilance et limitent la sensation de fatigue. Ces manifestations temporaires sont suivies d'une phase d'abattement, avec irritabilité, dépression, lassitude et souvent agressivité. Les sujets qui recourent régulièrement aux amphétamines font montre d'une activité maniaque, d'altérations du jugement, d'une augmentation de l'agressivité parfois accompagnée de passage à l'acte.

L'usage d'ecstasy altère les performances professionnelles ainsi que les performances routières notamment la fatigue insurmontable suivant la phase d'insomnie provoquée par la molécule.

\section{Opiacés}

Les effets psychiques des opiacés diffèrent selon leur utilisation.

En cas d'utilisation ponctuelle et à faible dose, on observe $(7,8,9)$ :

- une action analgésique accompagnée de sédation, 'somnolence et sommeil. La sédation de la douleur est due à l'augmentation du seuil de perception de celle-ci par suite de l'inhibition de la libération de substance $P$ (neuromédiateur spécifique des voies nerveuses contrôlant les sensations douloureuses) et de l'activation des systèmes inhibiteurs bloquant l'action des neurones de la douleur (voies ascendantes de la douleur). La morphine provoque donc un état d'indifférence aux stimulis douloureux ;

- une action dépressive sur les centres respiratoires pouvant conduire à des malaises ;

- une action psychodysleptique avec euphorie, voire dysphorie, exaltation de l'imagination et hallucinations ; 
- une hypotension et une bradycardie diminuant les performances du myocarde sans modifier le débit ( $d$ 'ou un risque d'arrêt cardiaque brutal) ;

- une constipation liée à une diminution du tonus et du péristaltisme intestinal.

Les effets psychophysiologiques de l'héroïne sont identiques à ceux de la morphine mais étant plus liposoluble, l'héroïne agit plus vite et plus intensément et la dépression respiratoire est plus marquée. On estime que son action est trois fois plus forte que celle de la morphine.

À fortes doses, l'héroïne provoque des bouffées délirantes, hallucinatoires et des phases d'excitation motrice parfois convulsives.

L'utilisation chronique d'héroïne peut entraîner une toxicomanie caractérisée par une dépendance physique et psychique ainsi que par une tolérance. Les troubles occasionnés peuvent rendre le sujet instable et plus ou moins dangereux pour les autres. Il s'agit :

- de perturbations continues du psychisme avec réactions paranoïaques ;

- de grandes variations de l'état de conscience ;

- et de comportements asociaux, agressifs et/ou dépressifs.

Tous ces effets induisent une perte de l'attention, des réflexes, de la réalité et de la conscience du danger. Leur utilisation est donc particulièrement contre-indiquée au cours de situations nécessitant concentration et dextérité.

Par ailleurs l'association héroïne + alcool est très dangereuse car elle augmente fortement la dépression centrale, donc la sédation et la vigilance.

Les effets indésirables de la codéine sont comparables à ceux des autres opiacés mais plus modérés et plus rares. La pholcodine, dérivée de la codéine, possède une action encore plus faible. Leur association à l'alcool entraîne une forte majoration des effets dépresseurs et sédatifs.

Bien qu'elle entraîne un syndrome de sevrage beaucoup moins important avec une dépression respiratoire plus faible, ce qui justifie son emploi en traitement de substitution, la buprénorphine possède une action analgésique considérée 25 à 40 fois supérieure à celle de la morphine. Son association à d'autres psychotropes dépresseurs du système nerveux central (alcool, benzodiazépines) est formellement déconseillée car elle entraîne l'endormissement et peut également provoquer des hallucinations.

La méthadone est un psychotrope également utilisé en traitement de substitution de la dépendance aux opiacés. Les effets sont comparables à ceux des autres opia- cés, mais ses propriétés euphorisantes sont faibles. Son action dépressive sur les centres respiratoires est plus élevée que celle de la morphine. Son utilisation répétée lui confère un effet sédatif marqué ainsi que l'apparition de mouvements réflexes exacerbés et une altération de la perception visuelle; tous ces effets s'avèrent peu compatibles avec une activité réclamant l'attention et la précision.

\section{Le cannabis}

Le chanvre est le plus souvent fumé seul ou en mélange avec du tabac sous forme de cigarettes ("joints") qui contiennent de 5 à $20 \mathrm{mg}$ de $\Delta-9$-THC dont 25 à $50 \%$ seront absorbés par les poumons. Il peut être parfois consommé par voie orale sous forme de pâtisseries (space cake). Les effets du cannabis, plus que pour toute autre drogue, varient d'un sujet à l'autre et pour le même sujet, d'une expérience à l'autre. Ils sont très sensibles à la personnalité, à l'état psychologique du moment et surtout à la dose ingérée.

Les effets du cannabis lors d'un usage occasionnel sont principalement (10) :

- des modifications de la perception du temps et des distances ;

- des perturbations de la mémoire à court terme ;

- des perturbations sensorielles : perturbation exacerbée des sons et surtout des modifications de la vision associées à une mydriase, une diplopie et un nystagmus ;

- des troubles thymiques et dissociatifs avec euphorie, anxiété, agressivité, dépersonnalisation avec disparition des inhibitions et indifférence vis à vis de l'environnement, une conscience accrue de soi;

- des hallucinations et délires exceptionnels mais possibles, notamment avec les nouveaux produits très concentrés en cannabinoïdes;

- une diminution des performances intellectuelles (baisse de la productivité et de la concentration avec une pensée fragmentaire), motrices et cognitives.

On peut noter aussi une décompensation psychotique se traduisant par un syndrome délirant organique dont le thème le plus fréquent est la persécution et associe anxiété, tremblements et incoordination motrice.

Lors d'un usage prolongé de cannabis, des crises d'angoisse aiguë peuvent survenir au cours desquelles un véritable état de panique s'installe. Par ailleurs, un syndrome amotivationnel est classiquement observé chez les usagers chroniques. Généralement lié à une imprégnation cannabique importante et ancienne, il associe un déficit de l'activité, une indifférence affective, une altération du fonctionnement intellectuel et un ralentissement de la pensée. Un usage régulier et important de cannabis favoriserait la survenue de la schizophrénie. 


\section{Conclusion}

Les données cliniques et pharmacologiques montrent que les psychotropes illicites ou stupéfiants peuvent exercer des effets néfastes sur la conduite automobile. Les opiacés entraînent une somnolence et un ralentissement de l'activité cérébrale. À l'inverse, la cocaïne et les amphétamines stimulent la vigilance et améliorent le temps de réaction mais parallèlement elles accroissent la prise de risques et peuvent rendre le conducteur agressif. Le cannabis altère la mémoire, l'humeur, les performances psychomotrices et cognitives de manière dose dépendante. L'utilisation de ces diverses substances est donc fortement préjudiciable à une conduite sécurisante.

\section{Références}

1. Charlier C., Verstraete A., Plomteux G. La législation dans les pays européens. In : Mura P., coordinateur. Alcool, médicaments, stupéfiants et conduite automobile. Paris : Elsevier, 1999, 215-32.

2. Julien RM. Ed. A Primer of Drug Action. New York : WH Freeman and Compagny, 1997.

3. Tassin JP. La place de la dopamine dans les processus de dépendance aux drogues. Bull. Acad. Natle Méd., 2002, 186, $2: 23-34$.

4. Nahas G., Neuropsycho-pharmacologie de la dépendance. In : Nahas G., Trouvé R. Toxicomanie Pharmacodépendance, Paris Masson Ed, 1988 ; 15-26.

5. Vasseur D., Chanson P. Drogues, savoir plus. Comité Français d'Education pour la santé Paris ,CFES, 1999 : $49 \mathrm{p}$.

6. Vinner E., Dehon B., Ghysel M-H., Lhermitte M. Les psychostimulants. In Mura P.,coordinateur, Alcool, médicaments, stupéfiants et conduite automobile. Paris Elsevier Option Bio, 1999 ; 97-128.

7. Pépin G., Chèze M. Les opiacés. In Mura P. coordinateur. Alcool, médicaments, stupéfiants et conduite automobile. Paris Elsevier-Option Bio, 1999 ; 75-95.

8. Pépin G. Opiacés et opioïdes. In Kintz P., coordinateur. Toxicologie et Pharmacologie Médicolégales. Paris Elsevier-Option Bio, 1998 ; 335-430.

9. Richard D., Pirot S., Senon J-L. Toxicomanies : Opiacés, LSD, Cocaïne, Amphétamines, Cannabis. In : Toxicologie, Paris, Le Moniteur Internat, Tome $1,2^{\text {cne éd, }}$ 1999 ; 277-311.

10. Mura P. Accidentologie et drogues illicites. Bull. Acad. Natle Méd. 2002 ; 186 : 73-85. 